\title{
Nebulized Ketamine: A Promising Approach for Managing Acute Pain in the Emergency Department
}

\author{
Meskine Amine ${ }^{1 *}$, Najout Hamza ${ }^{1}$, Moutawakil Mohamed ${ }^{1}$, Meziane Mohamed $^{1}$, Bensghir Mustapha $^{1}$
}

${ }^{1}$ Department of Anesthesia- Intensive Care, Mohammed-V Military Training Hospital, Faculty of Medicine and Pharmacy of Rabat, University Mohammed V, Rabat, Morocco

DOI: $10.36347 /$ sjmcr.2022.v10i01.006

| Received: 13.12.2021 | Accepted: 20.01.2022 | Published: 24.01.2022

*Corresponding author: Meskine Amine

Department of Anesthesia- Intensive Care, Mohammed-V Military Training Hospital, Faculty of Medicine and Pharmacy of Rabat, University Mohammed V, Rabat, Morocco

Abstract

Case Report

Acute severe pain is the most common condition for emergency consultations. opioids are the cornerstone of the treatment. However, they expose to various adverse effects. We describe a case of a 22-year-old woman with severe pain to her right hand, after a domestic fall, successfully managed by nebulized ketamine. Ketamine administration via nebulized route might be an attractive and non-invasive alternative to opioids use in acute pain management.

Keywords: Acute Pain; opioids; nebulized ketamine.

Copyright $(\mathcal{2 0 2 2}$ The Author(s): This is an open-access article distributed under the terms of the Creative Commons Attribution 4.0 International License (CC BY-NC 4.0) which permits unrestricted use, distribution, and reproduction in any medium for non-commercial use provided the original author and source are credited.

\section{INTRODUCTION}

Acute pain management in the emergency department is a top priority. The therapeutic approach must combine the best result in the shortest possible time with minimum complications. Opioids are the most commonly used, but they expose to several side effects. Recently, novel therapeutic strategies were described as an alternative to opioids use especially ketamine via nebulized route [1].

\section{CASE REPORT}

A 22-year-old women without any medical history was admitted to emergency department for traumatic hand injury after a domestic fall. She had a severe pain to her right hand after a fall with the patient landing on. she was unable to move her right wrist because of pain.

On examination, she was anxious and criedout of pain (7/10 on the numerical rating scale). She had a moderate swelling and tenderness to palpation at the metacarpal area. She had no ecchymosis or laceration and the neurovascular examination was normal. standard Radiographs were negative for acute dislocation or fracture.

Since the patient refused vascular access, we attempt ketamine via nebulized route through a nebulizer device at the dose of $0,5 \mathrm{mg} / \mathrm{kg}$ and were carful for any side effects requiring immediate cessation of nebulization. (The patient was aware of potential adverse events and approved the procedure).

The pain score decreased from 7 to 3 with in $30 \mathrm{~min}$. There after we decided to deliver a second dose of ketamine $(0,5 \mathrm{mg} / \mathrm{kg})$, which further reduced the pain from 3 to 0 on the numerical rating scale an hour after. No side effects were noted.

The patient, painless, was discharged 1 hour later with the final diagnosis of harmless wrist trauma and analgesia was supplied by oral paracetamol.

\section{DISCUSSION}

Acute Pain is the most common condition for emergency consultations. Opioids are the cornerstone of the treatment. However, they expose to a various side effects such as nausea, vomiting, urine retention, and respiratory depression. They also induce hyperalgesia causing increased analgesic requirements [2].

Recently, novel therapeutic strategies were described as an alternative to opioids use, such as locoregional analgesia blocks, modulating ion channels drugs, modulating enzymes drugs and ketamine [1].

Ketamine, commonly used in emergency department via intravenous or intra nasal route, is a 
dissociative anesthetic with analgesic and psychodysleptic effects. These outcomes are mediated through its non-competitive antagonist action on $\mathrm{N}$ methyl-D-aspartate/ glutamate receptor [3]. Ketamine, known as a safe drug, decreases pain by diminishing central sensitization, hyperalgesia, and nerve impulse transmission at the level of the spinal cord and central nervous system [4].

However, when intravenous access is inaccessible or mucosal atomization device is unreachable, nebulized routes may be used. A few reports have suggested that this route of use is both practical and reasonable for various reasons. It provides rapid, effective, and titratable analgesic delivery. It also minimizes analgesic toxicity and side effects. Further more, it improves overall management of painful conditions and reduces length of emergency department stay [5].

In the other hand, nebulized ketamine can expose to potential adverse events such as psychedelic effect, cardiac dysrhythmias, hypersalivation, hoarseness, oropharyngeal irritation, hypersalivation, nausea and vomiting [6].

In a case series, Drapkin $J$ et al described five patients who presented to the ED with acute painful conditions and treated with nebulized ketamine. They reported a decrease in pain from the baseline to $120 \mathrm{~min}$ with average change in pain score of 4.6 at $30 \mathrm{~min}, 6.4$ at $60 \mathrm{~min}$ and 7.8 at $120 \mathrm{~min}$. Only one patient experienced dizziness of modest intensity [7].

In a clinical randomized trial, Abdel-Ghaffar $H S$ et al compared the analgesic efficacy of nebulized ketamine ( 1 and $2 \mathrm{mg} / \mathrm{kg}$ ) administered 30min before general anesthesia in children undergoing elective tonsillectomy in comparison with IV ketamine $(0.5 \mathrm{mg} / \mathrm{kg})$ and saline placebo. They concluded that nebulized ketamine was effective for post-tonsillectomy pain relief and It can be considered as an effective alternative route to intravenous ketamine [8].

In our case the plausible mechanism of nebulized ketamine permitting the rapid management of the pain, besides its systemic action with analgesic property and rapid delivery, is the anxiolytic effect which potentiates analgesic effect.

\section{CONCLUSION}

Nebulized ketamine in our case shows that this uncommon way of use might be an attractive and non invasive alternative to opioids use in acute pain management. However further studies are required to confirm its efficiency and safety in this indication.

Funding: None.

Conflict of interest: None.

\section{REFERENCES}

1. Nowacka, A., \& Borczyk, M. (2019). Ketamine applications beyond anesthesia-A literature review. European journal of pharmacology, 860, 172547.

2. Yekkirala, A. S., Roberson, D. P., Bean, B. P., \& Woolf, C. J. (2017). Breaking barriers to novel analgesic drug development. Nature reviews Drug discovery, 16(8), 545-564.

3. Anis, N. A., Berry, S. C., Burton, N. R., \& Lodge, D. (1983). The dissociative anaesthetics, ketamine and phencyclidine, selectively reduce excitation of central mammalian neurones by $\mathrm{N}$-methylaspartate. British journal of pharmacology, 79(2), 565-575.

4. Gao, M., Rejaei, D., \& Liu, H. (2016). Ketamine use in current clinical practice. Acta Pharmacologica Sinica, 37(7), 865-872.

5. Thompson, J. P., \& Thompson, D. F. (2016). Nebulized fentanyl in acute pain: a systematic review. Annals of Pharmacotherapy, 50(10), 882891.

6. Allen, C. A., \& Ivester Jr, J. R. (2017). Ketamine for pain management-side effects \& potential adverse events. Pain Management Nursing, 18(6), 372-377.

7. Drapkin, J., Masoudi, A., Butt, M., Hossain, R., Likourezos, A., \& Motov, S. (2020). Administration of nebulized ketamine for managing acute pain in the emergency department: a case series. Clinical practice and cases in emergency medicine, 4(1), 16-20.

8. Abdel-Ghaffar, H. S., Abdel-Wahab, A. H., Roushdy, M. M., \& Osman, A. M. (2019). Uso preventivo de cetamina nebulizada para controle da dor após amigdalectomia em crianças: estudo randômico e controlado. Revista Brasileira de Anestesiologia, 69, 350-357. 